\title{
Classic Histology
}

National Cancer Institute

\section{Source}

National Cancer Institute. Classic Histology. NCI Thesaurus. Code C129470.

A term that refers to the histologic image of a lesion which carries all the characteristic morphologic findings which are necessary to define this lesion. 